Xue-Guo Liu* and Hui-Yan Pan

\title{
Crystal structure of $1,1^{\prime}$ -
}

(1,3-phenylenebis(methylene))bis(pyridin-1-ium) bis(1,2-dicyanoethene-1,2-dithiolato- $\left.\mathrm{K}^{2} S: S\right)$ palladium(II), $\mathrm{C}_{26} \mathrm{H}_{18} \mathrm{~N}_{6} \mathrm{PdS}_{4}$

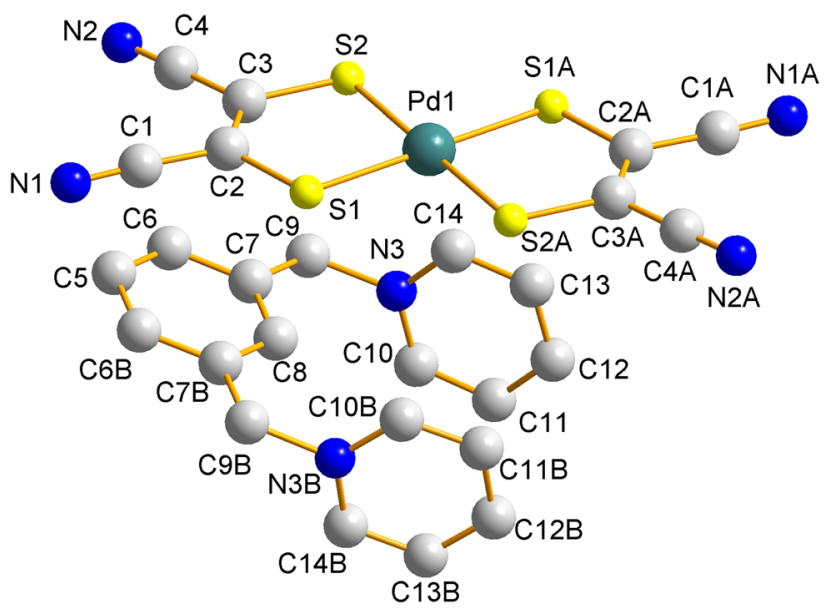

https://doi.org/10.1515/ncrs-2021-0292

Received July 17, 2021; accepted August 20, 2021;

published online October 1, 2021

\section{Abstract \\ $\mathrm{C}_{26} \mathrm{H}_{18} \mathrm{~N}_{6} \mathrm{PdS}_{4}$, monoclinic, $C 2 / c$ (no. 15), $a=16.658(2) \AA$, $b=10.398(3) \AA, c=17.284(2) \AA, \quad \beta=116.928(3)^{\circ}$, $V=2669.2(9) \AA^{3}, Z=4, R_{g t}(F)=0.0330, w R_{r e f}\left(F^{2}\right)=0.0912$, $\mathrm{T}=293(2) \mathrm{K}$}

\section{CCDC no.: 2104562}

The molecular structure is shown in the figure. Table 1 contains crystallographic data and Table 2 contains the list of the atoms including atomic coordinates and displacement parameters.

\footnotetext{
*Corresponding author: Xue-Guo Liu, School of Biology and Chemica Engineering, Nanyang Institute of Technology, Nanyang 473004 , China, E-mail: 512460415@qq.com. https://orcid.org/0000-00016331-9025

Hui-Yan Pan, School of Biology and Chemical Engineering, Nanyang Institute of Technology, Nanyang 473004, China
}

Table 1: Data collection and handling.

\begin{tabular}{ll}
\hline Crystal: & Brown block \\
Size: & $0.24 \times 0.22 \times 0.20 \mathrm{~mm}$ \\
Wavelength: & Mo $K \alpha$ radiation $(0.71073 \AA$ A $)$ \\
$\mu:$ & $1.04 \mathrm{~mm}^{-1}$ \\
Diffractometer, scan mode: & Bruker APEX II \\
$\theta_{\text {max }}$, completeness: & $27.8^{\circ},>99 \%$ \\
$N(h k l)_{\text {measured }}, N(h k l)_{\text {unique }}, R_{\text {int: }}:$ & $8293,3130,0.022$ \\
Criterion for $I_{\text {obs }}, N(h k l)_{\mathrm{gt}}:$ & $I_{\text {obs }}>2 \sigma\left(I_{\text {obs }}\right), 2764$ \\
$N(\text { param })_{\text {refined }}:$ & 170 \\
Programs: & SHELX [1], Bruker [2] \\
\hline
\end{tabular}

\section{Source of material}

All reagents and chemicals were purchased from commercial sources and used without further purification. The starting materials disodium maleonitriledithiolate and 1,3-bis(methylpyridinium benzene) bromide were synthesized following the literature procedures $[3,4]$. An aqueous solution $(10 \mathrm{~mL})$ of 1,3-bis(methylpyridinium benzene) bromide $(0.0842 \mathrm{~g}, 0.2 \mathrm{mmol})$ was added slowly to an aqueous solution $(20 \mathrm{~mL})$ of disodium maleonitriledithiolate $(0.0741 \mathrm{~g}, 0.4 \mathrm{mmol})$ and $\mathrm{K}_{2} \mathrm{PdCl}_{4}(0.0982 \mathrm{~g}$, $0.3 \mathrm{mmol}$ ), and the mixture was stirred at room temperature for several minutes. A brown precipitate was filtered off, washed by water and dried under vacuum. The precipitate was dissolved in dimethylformamide with ether diffusion. Two weeks later brown crystals were obtained.

\section{Experimental details}

Absorption corrections were applied using the multi-scan method. Hydrogen atoms were located in difference electron density maps, and treated as riding atoms. The $U_{\text {iso }}$ values of the hydrogen atoms were set to $1.2 U_{\mathrm{eq}}(\mathrm{C})$.

\section{Comment}

Complexes have been extensively studied for a long time, since potentially they could be candidates for magne 
Table 2: Fractional atomic coordinates and isotropic or equivalent isotropic displacement parameters $\left(\AA^{2}\right)$.

\begin{tabular}{lrrrr}
\hline Atom & $\boldsymbol{x}$ & $\boldsymbol{y}$ & $\boldsymbol{z}$ & $\boldsymbol{U}_{\text {iso }} / \boldsymbol{U}_{\text {eq }}$ \\
\hline C1 & $0.12172(17)$ & $0.6429(2)$ & $0.46994(16)$ & $0.0407(5)$ \\
C2 & $0.17154(16)$ & $0.5313(2)$ & $0.47022(15)$ & $0.0371(5)$ \\
C3 & $0.22182(16)$ & $0.5315(2)$ & $0.42544(15)$ & $0.0366(5)$ \\
C4 & $0.22507(17)$ & $0.6431(2)$ & $0.37937(16)$ & $0.0396(5)$ \\
C5 & 0.000000 & $0.6132(3)$ & 0.250000 & $0.0443(8)$ \\
H5 & -0.000001 & 0.702600 & 0.249999 & $0.053^{*}$ \\
C6 & $0.04345(17)$ & $0.5482(2)$ & $0.21186(16)$ & $0.0392(5)$ \\
H6 & 0.073089 & 0.592816 & 0.185729 & $0.047^{*}$ \\
C7 & $0.04323(17)$ & $0.4165(2)$ & $0.21221(16)$ & $0.0380(5)$ \\
C8 & 0.000000 & $0.3518(3)$ & 0.250000 & $0.0416(8)$ \\
H8 & -0.000001 & 0.262399 & 0.249999 & $0.050^{*}$ \\
C9 & $0.0878(2)$ & $0.3488(2)$ & $0.16342(19)$ & $0.0478(6)$ \\
H9A & 0.055707 & 0.370588 & 0.102325 & $0.057^{*}$ \\
H9B & 0.149166 & 0.379364 & 0.185013 & $0.057^{*}$ \\
C10 & $0.02345(19)$ & $0.1362(3)$ & $0.11344(17)$ & $0.0470(6)$ \\
H10 & -0.019892 & 0.175013 & 0.063476 & $0.056^{*}$ \\
C11 & $0.0204(2)$ & $0.0086(3)$ & $0.1256(2)$ & $0.0549(7)$ \\
H11 & -0.025135 & -0.040860 & 0.083890 & $0.066^{*}$ \\
C12 & $0.0846(2)$ & $-0.0484(2)$ & $0.1994(2)$ & $0.0592(7)$ \\
H12 & 0.082759 & -0.136544 & 0.207764 & $0.071^{*}$ \\
C13 & $0.1496(2)$ & $0.0233(3)$ & $0.2592(2)$ & $0.0532(7)$ \\
H13 & 0.193265 & -0.014487 & 0.309464 & $0.064^{*}$ \\
C14 & $0.15167(18)$ & $0.1517(2)$ & $0.24617(16)$ & $0.0449(6)$ \\
H14 & 0.196659 & 0.202211 & 0.287649 & $0.054^{*}$ \\
N1 & $0.07857(18)$ & $0.7308(2)$ & $0.46888(16)$ & $0.0470(6)$ \\
N2 & $0.22659(17)$ & $0.73123(19)$ & $0.33957(16)$ & $0.0461(6)$ \\
N3 & $0.08861(15)$ & $0.2060(2)$ & $0.17322(14)$ & $0.0392(5)$ \\
Pd1 & 0.250000 & 0.250000 & 0.500000 & $0.03578(11)$ \\
S1 & $0.16297(4)$ & $0.39845(5)$ & $0.52664(4)$ & $0.03943(16)$ \\
S2 & $0.28296(4)$ & $0.39979(5)$ & $0.42148(4)$ & $0.03920(16)$ \\
\hline & & & &
\end{tabular}

tism, luminescence and catalysis as well as pharmaceutical aspect, etc. [5-9]. Among these numerous complexes those with the maleonitriledithiolate (mnt) ligand have been extensively studied. Varieties of maleonitriledithiolate complexes have shown unusual optical, electrical, magnetic properties and so on [10-14]. This contribution is part of our present project on the design and synthesis of complexes based on the mnt ligand [15, 16]. Herein, we choose 1,3-bis(methylpyridinium benzene) as organic divalent cation $[1,3-\mathrm{BMPB}]^{2+}$, to stabilize the anionic palladium(II) complex. X-ray crystallography analyses reveal that the title complex shows 3D framework linked through extensive hydrogen bonding interactions.

The asymmetric contains one half of the $\left[\operatorname{Pd}(\mathrm{mnt})_{2}\right]^{2-}$ dianion and of the 1,3-bis(methylpyridinium benzene) dication (see the Figure). The $\mathrm{Pd}(\mathrm{II})$ is coordinated by four $\mathrm{S}$ atoms of two maleonitriledithiolate ligands, and exhibits the expected square-planar coordination geometry. The Pd1-S bond lengths and S-Pd1-S bond angles are in 2.2900(7)-2.3025(7) A and 89.76(3)-180', respectively. All bond lengths and bond angles are consistent with the a reported complexes [17]. In comparison with a reported complex [15], the anions and cations do not form completely separated columns but cross each other. This difference may be due to cation structure hindrance. Some weak $\mathrm{C}-\mathrm{H} \cdots \mathrm{N}$ interactions are observed between anions and cations [18].

Author contributions: All the authors have accepted responsibility for the entire content of this submitted manuscript and approved submission.

Research funding: This study was financially support by Scientific and Technological Research Projects of Henan Province (182102311077).

Conflict of interest statement: The authors declare no conflicts of interest regarding this article.

\section{References}

1. Sheldrick G. M. Crystal structure refinement with SHELXL. Acta Crystallogr. 2015, C71, 3-8.

2. Bruker. SMART APEX-II CCD Bruker AXS Inc.: Madison, WI, USA, 2006.

3. Simmons H. E., Blomstrom D. C., Vest R. D. Thiacyanocarbons. II. chemistry of disodium dimercaptomaleonitrile. J. Am. Chem. Soc. 1962, 84, 4756-4771.

4. Musilek K., Komloova M., Zavadova V., Holas O., Hrabinova M., Pohanka M., Dohnal V., Nachon F., Dolezal M., Kuca K., Jung Y. S. Preparation and in vitro screening of symmetrical bispyridinium cholinesterase inhibitors bearing different connecting linkageinitial study for Myasthenia gravis implications. Bioorg. Med. Chem. Lett 2010, 20, 1763-1766.

5. Kurmoo M. Magnetic metal-organic frameworks. Chem. Soc. Rev. 2009, 38, 1353-1379.

6. Zou H. H., Meng T., Chen Q., Zhang Y. Q., Wang H. L., Li B., Wang K., Chen Z. L., Liang F. P. Bifunctional mononuclear dysprosium complexes: single-ion magnet behaviors and antitumor activities. Inorg. Chem. 2019, 58, 2286-2298.

7. Allendorf M. D., Bauer C. A., Bhakta R. K., Houk R. J. T. Luminescent metal-organic frameworks. Chem. Soc. Rev. 2009, 38, 1330-1352.

8. Lee J., Farha O. K., Roberts J., Scheidt K. A., Nguyen S. T., Hupp J. T. Metal-organic framework materials as catalysts. Chem. Soc. Rev. 2009, 38, 1450-1459.

9. Fan H. T., Xing X. J., Yang Y. H., Li B., Wang C. C., Qiu D. F. Triple function nanocomposites of porous silica-CoFe $\mathrm{O}_{4}$-MWCNTs as a carrier for $\mathrm{pH}$-sensitive anti-cancer drug controlled delivery. Dalton Trans. 2017, 46, 14831-14838.

10. Coomber A. T., Beljonne D., Friend R. H., Brédas J. L., Charlton A., Robertson N., Underbill A. E., Kurmoo M., Day P. Intermolecular 
interactions in the molecular ferromagnetic $\mathrm{NH}_{4} \mathrm{Ni}(\mathrm{mnt})_{2} \cdot \mathrm{H}_{2} \mathrm{O}$. Nature 1996, 380, 144-146.

11. Robertson N., Cronin L. Metal bis-1,2-dithiolene complexes in conducting or magnetic crystalline assemblies. Coord. Chem. Rev. 2002, 227, 93-127.

12. Duan H. B., Ren X. M., Meng Q. J. One-dimensional (1D) $\left[\mathrm{Ni}(\mathrm{mnt})_{2}\right]^{-}$ based spin-Peierls-like complexes: structural, magnetic and transition properties. Coord. Chem. Rev. 2010, 254, 1509-1522.

13. Wang P. Crystal structure of $\mathrm{Mn}(\mathrm{II})\left(\mathrm{H}_{2} \mathrm{O}\right)(2,13$-dimethyl-3,6,9,12,18pentaazabicyclo[12.3.1]octadeca-1(18),2,12,14,16-pentaene)-bis(maleonitriledithiolate)nickel(II), $\mathrm{C}_{23} \mathrm{H}_{25} \mathrm{MnN}_{9} \mathrm{NiOS}_{4}$. Z. Kristallogr. N. Cryst. Struct. 2012, 227, 511-512.

14. Yan W. H., Ji E. Y., Shen M. L., Li Z. Y., Li X., Xu X. L. Two ion-pair complexes constructed by $\left[\mathrm{M}(\mathrm{mnt})_{2}\right]^{n-}(\mathrm{M}=\mathrm{Ni}, \mathrm{Co}, \mathrm{mnt}=$ maleonitriledithiolate): syntheses, characterization and thermal stability. Chin. J. Struct. Chem. 2015, 34, 306-312.
15. Yan W. H., Pan H. Y. Crystal structure of 1,4-bis(methylpyridinium benzene) bis(1,2-dicyanoethene-1,2-dithiolato- $\kappa^{2} S: S$ )nickel(II), $\mathrm{C}_{26} \mathrm{H}_{18} \mathrm{~N}_{6} \mathrm{NiS}_{4}$. Z. Kristallogr. N. Cryst. Struct. 2021, 236, 107-108.

16. Yan W. H., Liu X. G., Shen M. L., Pan H. Y. Crystal structure of 1,1(1,4-phenylenebis(methylene))bis(pyridin-1-ium) bis (1,2-dicyanoethene-1,2-dithiolato- $\mathrm{k}^{2} S: S$ ) zinc(II), $\mathrm{C}_{26} \mathrm{H}_{18} \mathrm{~N}_{6} \mathrm{ZnS}_{4}$,. Z. Kristallogr. N. Cryst. Struct. 2021, 236, 533-535.

17. Pilia L., Espa D., Barsella A., Fort A., Makedonas C., Marchio L., Mercuri M. L., Serpe A., Mitsopoulou C. A., Deplano P. Combined experimental and theoretical study on redox-active $\mathrm{d}^{8}$ metal dithione- dithiolato complexes showing molecular second-order nonlinear optical activity. Inorg. Chem. 2011, 50, 10015-10027.

18. Jeffrey G. A. Hydrogen-bonding: an update. Crystallogr. Rev. 2003, 9, 135-176. 